\title{
Monitoring of solenoid parameters based on neural networks and optical fiber squeezer for solenoid valves diagnosis
}

\author{
Abdallah Zahidi ${ }^{1}$, Said Amrane ${ }^{2}$, Nawfel Azami ${ }^{3}$, Naoual Nasser ${ }^{4}$ \\ ${ }^{1,2,3}$ INPT Optics Lab, National Institute of Posts and Telecommunications, Rabat, Morocco \\ ${ }^{4}$ LDEDS, Faculties of Science and Technology, Hassan University $1^{\text {st }}$, Settat, Morocco
}

\begin{tabular}{l}
\hline Article Info \\
\hline Article history: \\
Received Apr 4, 2020 \\
Revised Aug 11, 2020 \\
Accepted Sep 30, 2020 \\
\hline
\end{tabular}

Keywords:

EMS

Fluctuations

Monitoring

Neural Networks

Polarization squeezer

\begin{abstract}
As crucial parts of various engineering systems, solenoid valves (SVs) operated by electromagnetic solenoid (EMS) are of great importance and their failure may lead to cause unexpected casualties. This failure, characterized by a degradation of the performances of the SVs, could be due to a fluctuations in the EMS parameters. These fluctuations are essentially attributed to the changes in the spring constant, coefficient of friction, inductance, and the resistance of the coil. Preventive maintenance by controlling and monitoring these parameters is necessary to avoid eventual failure of these actuators. The authors propose a new methodology for the functional diagnosis of electromagnetic solenoids (EMS) used in hydraulic systems. The proposed method monitors online the electrical and mechanical parameters varying over time by using articial neural networks algorithm coupled with an optical fiber polarization squeezer based on EMS for polarization scrambling. First, the MATLAB/Simulink model is proposed to analyze the effect of the parameters on the dynamic EMS model. The result of this simulation is used for training the neural network. Then a simulation is proposed using the neural net tting toolbox to determine the solenoid parameters (Resistance of the coil R, stiffness $\mathrm{K}$ and coefficient of friction $\mathrm{B}$ of the spring) from the coefficients of the transfer function, established from the model step response. Future work will include not only diagnosing failure modes, but also predicting the remaining life based on the results of monitoring.
\end{abstract}

This is an open access article under the CC BY-SA license.

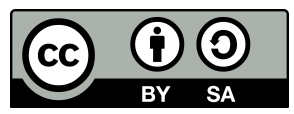

\section{Corresponding Author:}

Abedallah Zahidi

National Institute of Posts and Telecommunications

Avenue Allal Al Fassi, Rabat, Morocco

Email: zahidiabdo@yahoo.fr

\section{INTRODUCTION}

In electromechanical solenoids (EMS), also called electromagnetic devices (EMD), driven by a control solenoid, the armature is positioned by balancing the electromagnetic force against that of a return spring. They are relatively an inexpensive construction [1], have a simple design and control circuit, require little energy for control, are highly reliable [2], these electromagnetic actuators are used in wide range of modern industrial equipment such as digital actuator arrays [3], vehicle vibration control systems [4], gas valve [5], robotic manipulators [6], positioners [7], anti-braking systems [8], and polarization controllers where solenoids are used as mechanical actuator on the fiber to adjust the output light power [9]. In hydraulic systems, these electromagnetic actuators are commonly used in three basic categories to actuate hydraulic control valves directional- 
control, flow-control, and pressure-control. Directional-control valves are used to connect and isolate hydraulic passages by simply opening and closing a communication path. Flow-control valves allow variable flow rate control to a component. Finally, pressure-control valves regulate variable pressure to a hydraulic component [10].

Analysis of EMS purposes show that to improve the safety and the performance of the EMS based hydraulic system, the regular maintenance strategy of these electromechanical solenoids surely reduces failure rate of the system; however, it brings high maintenance cost [11]. It should be highlighted that emergency modes of EMDs are not only results of faults of their various elements or incorrect personnel activities during EMD manufacturing and operation, they are also possible during a normal operation due to the wearing of mating surfaces of friction assemblies or/and fluctuation in its parameters [12, 13].

Therefore, it is necessary to develop an effective approach for EMS diagnostics and operation controls in order to map the failure and the reliability of the EMS based on the solenoid valves. The diagnostic results could be used to estimate the health condition of the solenoid valves and predict their remaining useful life. Additionally, the solenoid valves could get timely repaired or replaced before their potential failure causes any system breakdown.

\section{PROBLEM STATEMENT}

Despite the high reliability and the excellent performance of solenoid valves in various applications, their failure may result in severe system crash, signficant casualties and economic losses, especially in safetyfirst fields, such as railway braking system, aviation engine, and nuclear power plants [14]. However, as stated in some previous works $[15,16]$, the EMS dynamic in these devices is governed by an electromagnetic force that increases greatly when the air gap is near zero. This nonlinear behavior, together with physical bounds that limit the motion, causes EMS of valves to be subject to strong shocks and wear that often result in early failures [17], these failures, which affects the dynamic response [18], may be due to a fluctuation in the EMS structure parameters [19] or the electrical and mechanical parameters of the EMS (variations in inductance and resistor of the coil, changes in spring constant and coefficient of friction) [20,21]. Other studies have shown a deterioration in the performance of EMS based system in presence of parametric variation [12, 13], even for the best control solutions [20]. Other literatures have also revealed that failure of solenoid valves can also occur gradually due to coil burnout of EMS related to mains voltage and frequency, spring force [22, 23]; and that the resistance of the coil might be a source of themo-mechanical failure of the solenoid valve [24]. In addition, the above-mentioned literature provides the parameters which characterize the failure of the solenoid valves. This information can then be used to design a model to map the failure. Approaches based on signal processing [25] and machine learning [26] have been proposed in the literature to diagnose the state of solenoid valves leading to the development of a sensor to detect anomalies [27] or a method for grouping failures [26]. None of these approaches gives a physical explanation of the failure modes related to the solenoid parameters [28]; Other models based on the movement of the armature and Foucault current [2] and the EMD winding current curve appearing with the movement of the armature [29, 30] have been developed for diagnosis, but none of these models does treat electrical and mechanical parameters as a source of failure. Morever, most control approaches are using signals such as the coil current or voltage of solenoid to monitor the parameters; yet, the main problems in such approaches are that the detected signals are prone to interference and difcult to obtain [31]. Other works have been limited to the estimation and identication of a single solenoid parameter [32, 22]. In this work, the authors have developed a new approach for the diagnosis of an EMS actuator in solenoid valve. The proposed approach is based on a new method based on optical fiber polarization controller signal feedback coupled with articial neural networks model (ANN) for monitoring the electrical and mechanical EMS parameters (resistence of coil R, and K, B respectively the stiffness and the coefficient of friction of the spring), considered in this approach as health indices to characterize the failure of the solenoid valve. NN has the advantages of controlling complex and non-linear systems [33,34], has high accuracy of prediction capability [35], and it is of great importance to find the high speed electromagnetic switching valve [36].

\section{METHODOLOGY OF STUDY}

This paper proposes a new methodology using optical fiber polarization controller signal feedback coupled with an articial neural networks model (ANN) for monitoring the solenoid parameters and predicting its performance. Figure 1 shows the proposed monitoring process. 


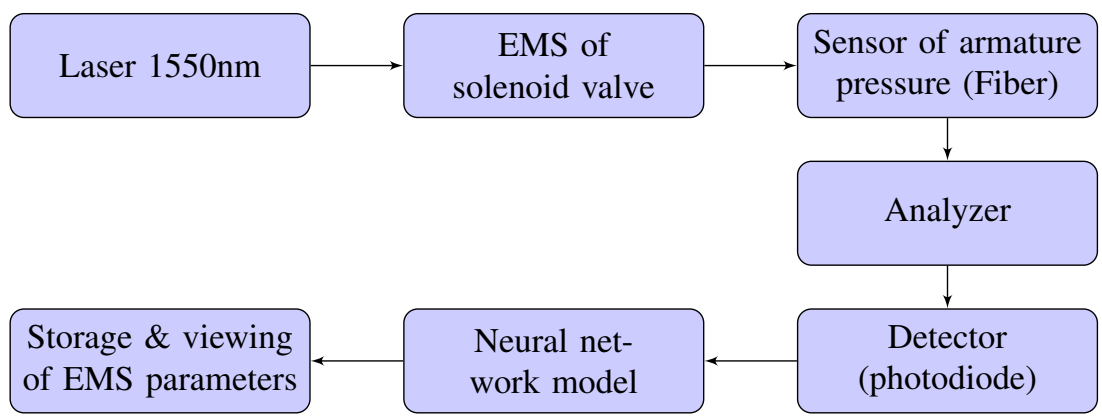

Figure 1. Block diagram of monitoring process

In the proposed structure, the optical fiber polarization controller is based on an EMS and an optical fiber used as a mechanical force sensor of the EMS armature. This force induces an optical birefringence that modifies the polarization of the light [9]. The variation of the polarization of the light is reflected by the variation of the light intensity detected by a photodiode placed at the output of the optical fiber, In the first step, a mathematical model is proposed to obtain the response of the system. Then, this model is identied from this response using function tfest of MATLAB/Simulink [37] to determine the transfer function coefficient of the system. In the second step, the effect of solenoid parameters variation on the transfer function coefficient is analysed. This method uses simulation electromagnetic fiber squeezer based polarization controller with function tfest and the simulation results are stored in a text file that will be used for neural networks training. In the last step, the neural networks model is proposed to estabish the solenoid parameters from the coefficients of the transfer function set from the step response of the fiber squeezer. Finally, to check the efficiency of the proposed model, a prediction error is calculated. The result of the simulation shows that this optical fiber squeezer coupled with the neural network model is very efficient to monitor the EMS parameters. The results of monitoring will be used in a future work to estimate the remaining life of the solenoid valve.

\section{BUILDING THE SIMULINK MODEL}

\subsection{Structure and equations of electromagnetic fiber squeezer} Figure 2 .

The EMS is the electromagnetic actuator that exerts the pressure on the fiber. Its structure is shown in

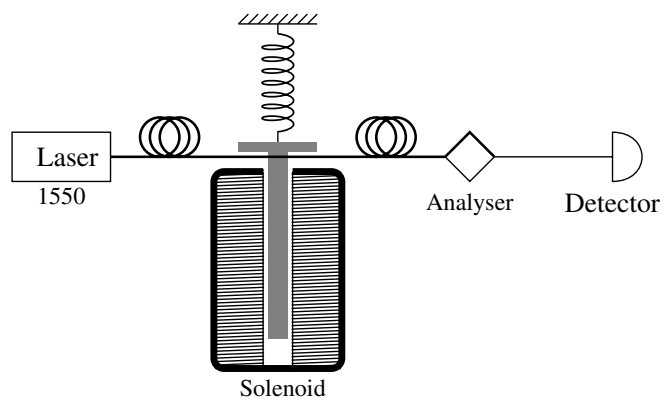

Figure 2. Scheme of using the electromagnetic fiber squeezer

The magnitude of the phase difference of two polarized light along the squeezing axis and its orthogonal axis can be expressed as [38]:

$$
\delta=6 e-5 \frac{F}{\lambda d}
$$

and the light power $P s$ at the output of the polarization analyzer according to scheme of Figure 2 is:

$$
P_{s}=E^{2}
$$


where

$$
\begin{gathered}
E=A \cdot \frac{1}{2} \cdot E \\
A=\left(\begin{array}{cc}
e^{j\left(\phi_{m}+\frac{\delta}{2}\right)} & 0 \\
0 & e^{j\left(\phi_{m}-\frac{\delta}{2}\right)}
\end{array}\right) \\
P_{s}=E^{2}=\frac{P_{0}}{2}(1+\cos \delta)
\end{gathered}
$$

where $P_{0}$ is the input light intensity.

\subsection{Mathematical model of EMS}

The solenoid refers to the electromagnetic actuator. It is used to exert pressure on the fiber. Its structure is shown in Figure 3.

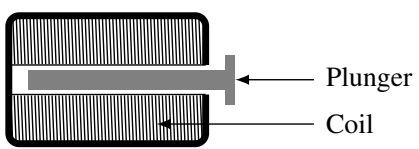

Figure 3. Cross section of EMS

The mathematical model of EMS is given [39] by expression as (6):

$$
m \frac{d^{2} x(t)}{d t^{2}}+B \frac{d x(t)}{d t}+K x(t)=\frac{\mu_{0} \mu_{r} N^{2} A I^{2}(t)}{2\left(x_{0}-x(t)\right)^{2}}
$$

where: $x(t)$ : Displacement of the armature in $(\mathrm{m}), I(t)$ : The electromagnet coil current in(A), $A$ : the cross sectional area of the coil in $\left(\mathrm{m}^{2}\right), N$ : the number of the turns of the coil, $\mu_{0}$ : Permeability of the free space in $(\mathrm{H} / \mathrm{m}), \mu_{r}$ : Relative Permeability of the dielectric materiel between the coil and armature, $x_{0}$ : The initial air gap between the armature and the backside of the frame in $(\mathrm{m}), m$ : Masse of the armature in $(\mathrm{Kg}), K$ : is the stiffness of the spring in $(\mathrm{N} / \mathrm{m})$ and $B$ : System damping coefficient in (N.s/m).

The equation of the electrical circuit is as (7) and (8):

$$
\begin{gathered}
u=R i(t)+\frac{d}{d t}[L(x) \cdot i(t)] \\
u=R i(t)+L(x) \frac{d i(t)}{d t}+i(t) \frac{d L(x)}{d t}
\end{gathered}
$$

$R$ is the series resistance of the EMS coil and $L(x)$ is the inductance of the coil that depends of the air gap [39]:

$$
L(x)=\frac{\mu_{0} \mu_{r}}{x_{0}-x(t)}
$$

The balance equation of the force acting on the fiber is expressed as [40]

$$
m \frac{d^{2} x(t)}{d t^{2}}=F-K\left(x(t)-x_{0}\right)-B \frac{d x(t)}{d t}
$$

$F$ is the force produced by the magnetic field and it be derived knowing that magnetic system is linear and that current was kept constant

$$
F=\frac{d w_{t}}{d x}=\frac{i^{2}}{2} \frac{d L(x)}{d x}=\frac{i^{2}}{2} \frac{a L^{\prime}}{(a+x)^{2}}
$$


where $L^{\prime}=\frac{\mu_{0} \pi a d N^{2}}{g}$ and $a, d, g$ parameters depending on the EMS. from (8) we can write

$$
\frac{d i}{d t}=\frac{1}{L(x)}\left(u-R i-i \frac{d L(x)}{d x} \frac{d x}{d t}\right)
$$

from (10) we can write

$$
\frac{d^{2} x}{d t^{2}}=\frac{1}{m}\left(F-K\left(x-x_{0}\right)-B \frac{d x}{d t}\right)
$$

Both (1) and (5) are used to write:

$$
P_{s}=\frac{P_{0}}{2}\left(1+\cos \left(6 e-5 \frac{F}{\lambda d}\right)\right)
$$

\subsection{Simulink model}

The system model has been implemented in versatile software MATLAB which is widely used in control engineering around the word. This simulation is used to effectively determine the best performance of the dynamic response in the output light intensity. The electrical model Simulink which models (12) is represented in Figure 4(a); while, the Simulink mechanical model for (13) is illustrated in Figure 4(b). This model depends on the intrinsic parameters of the EMS: the mass $\mathrm{m}$ of the armature, the coefficient of friction $B$, the stiffness of the ressort $K$, the resistance and the inductance of the coil $(R, L)$. The Figure 4(c) represents the optical Simulink model for (14).

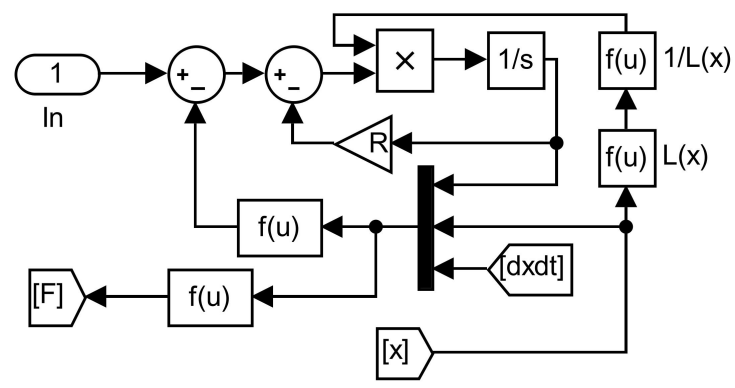

(a)

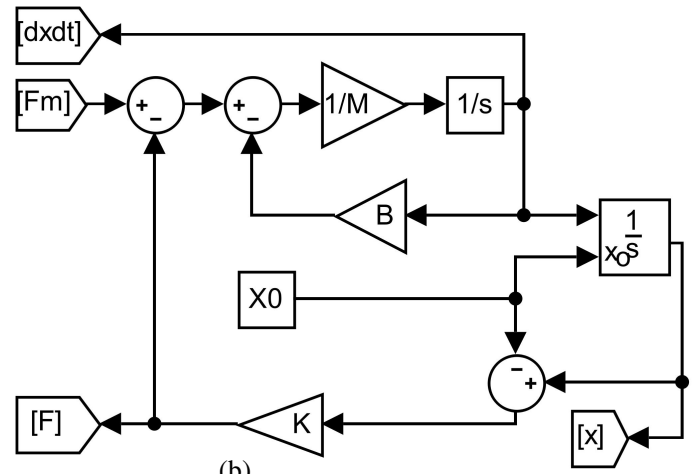

(b)

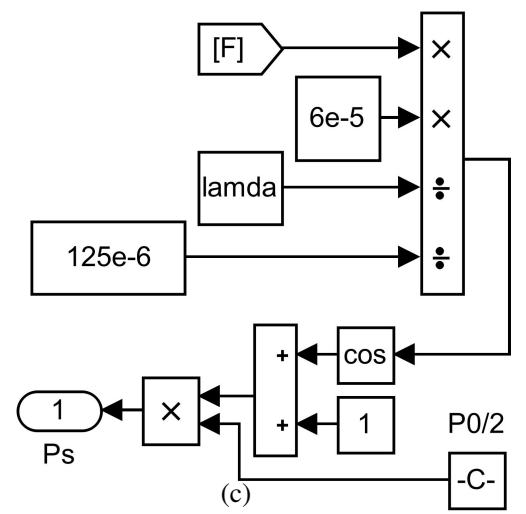

Figure 4. Simulink models, (a) Electrical model, (b) Mechanical model, (c) Optical model 


\section{PRINCIPE OF SIMULATION}

\subsection{Monitoring process flowchart}

To predict and monitor the EMS parameters, the coefficients of the transfer function obtained from the step response of the fiber squeezer based on the EMS are used as input of the NN model. The parameters solenoid R, B and $\mathrm{K}$ are expected as outputs. The flowchart of the monitoring process is shown in Figure 5. Figure 6 proposes the architecture of the ANN model and Table 1 illustrates an example of identification results used for the ANN training.

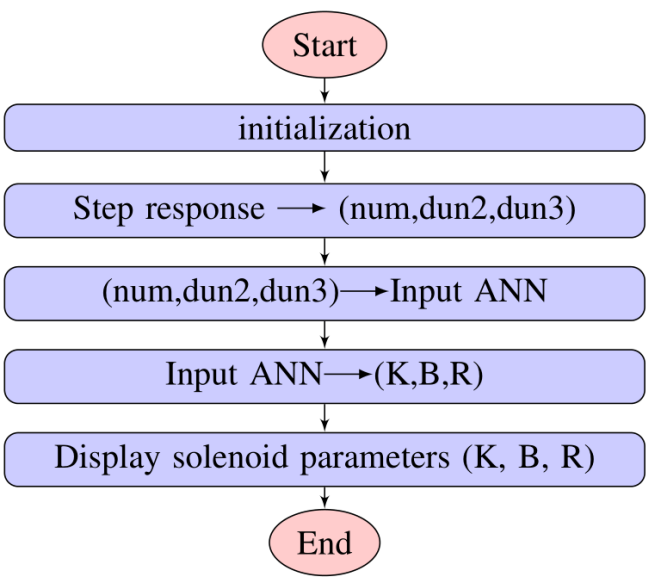

Figure 5. Monitoring process flowchart

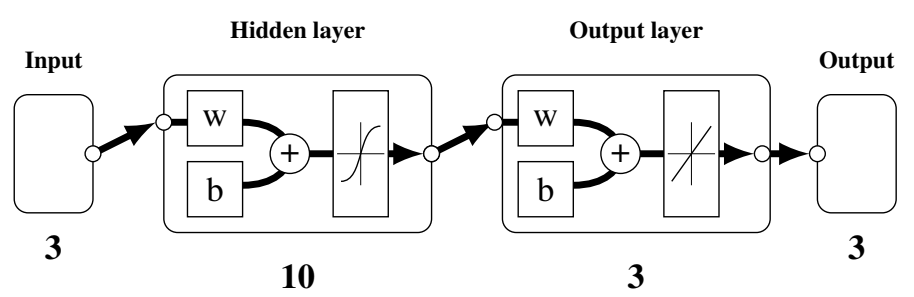

Figure 6. Neural network architecture

Table 1. Identification result for neural network training

\begin{tabular}{cccccccc}
\hline Indice & $\mathrm{K}(\mathrm{N} / \mathrm{m})$ & $\mathrm{B}(\mathrm{Ns} / \mathrm{m})$ & $\mathrm{R}(\Omega)$ & Num & Dun1 & Dun2 & Dun3 \\
\hline 1 & 2998.5692 & 3.2177 & 11.8536 & 114.7376 & 1 & 16.2331 & 15095.4567 \\
2 & 2168.9635 & 2.3559 & 12.8581 & 70.5311 & 1 & 11.8918 & 10931.6472 \\
3 & 2521.0054 & 2.1333 & 13.6778 & 72.4483 & 1 & 10.7603 & 12681.9928 \\
\hline
\end{tabular}

\subsection{Neural networks architecture}

The NN inputs consist of a matrix of order $10000 \times 3$, where each line represents a set of coefficients of the transfer function num, dun2 and dun3. On the other hand, the outputs are the elements of a matrix of the same order as the inputs where each line exemplifies a set of solenoid parameters (K, B and R). The structure also contains 10 hidden layers chosen by default with the sigmoid function as activation function and 3 output layers with a linear activation function as shown in Figure 6.

\subsection{Neural networks training}

\subsubsection{Identification with variable parameters for $\mathrm{NN}$ training}

First, we propose a mathematical model that is used to obtain the response of the system. Then, this model is identified from this response using identification function tfest whose syntax is sys=tfest(data, $\mathrm{np}, \mathrm{nz}$ ). This function is used to estimate a transfer function containing nz zeros and np poles from the index response 
(data) of the Simulink model described in paragraph 4.3. The resulting transfer function has the coefficient (num, dun2, dun3), and can be expressed as (15):

$$
T f=\frac{n u m}{s^{2}+d u n 2 s+d u n 3}
$$

Secondly, in order to obtain the system transfer function for different values of the solenoid parameters, $\mathrm{K}, \mathrm{B}$ and $\mathrm{R}$ are varied while while keeping $\mathrm{m}=200 \mathrm{~g}$ and $\mathrm{L}=20 \mathrm{mH}$ since they are not likely to vary during long-term operation of the solenoid. The variation interval of $\mathrm{K}$ is 1000 to $3000 \mathrm{~N} / \mathrm{m} ; \mathrm{R}$ is 10 to $15 \Omega$ and $\mathrm{B}$ is 2 to $4 \mathrm{~N} . \mathrm{s} / \mathrm{m}$. The inputs of the Simulink model are a matrix of three columns and $\mathrm{n}$ rows of random value of solenoid parameter. The random variation between the max and min values of each of the three parameters (K, $\mathrm{B}$ and $\mathrm{R})$ is obtained by using the function rand (n,1) whose syntax is as (16):

$$
\text { parameter }=(\max -\min ) * \operatorname{rand}(n, 1)+\min
$$

where parameter is $\mathrm{K}, \mathrm{R}$ or $\mathrm{B}$, and $\mathrm{n}=10$ 000. The following Figure 7 represents the flowchart which allows to obtain the dataset and Table 1 shows an example of the text file results obtained. The results of this simulation are used as data (num, dun2, dun3) and target (K, B, R) for training the neural network model as shown in section 5.3.2.

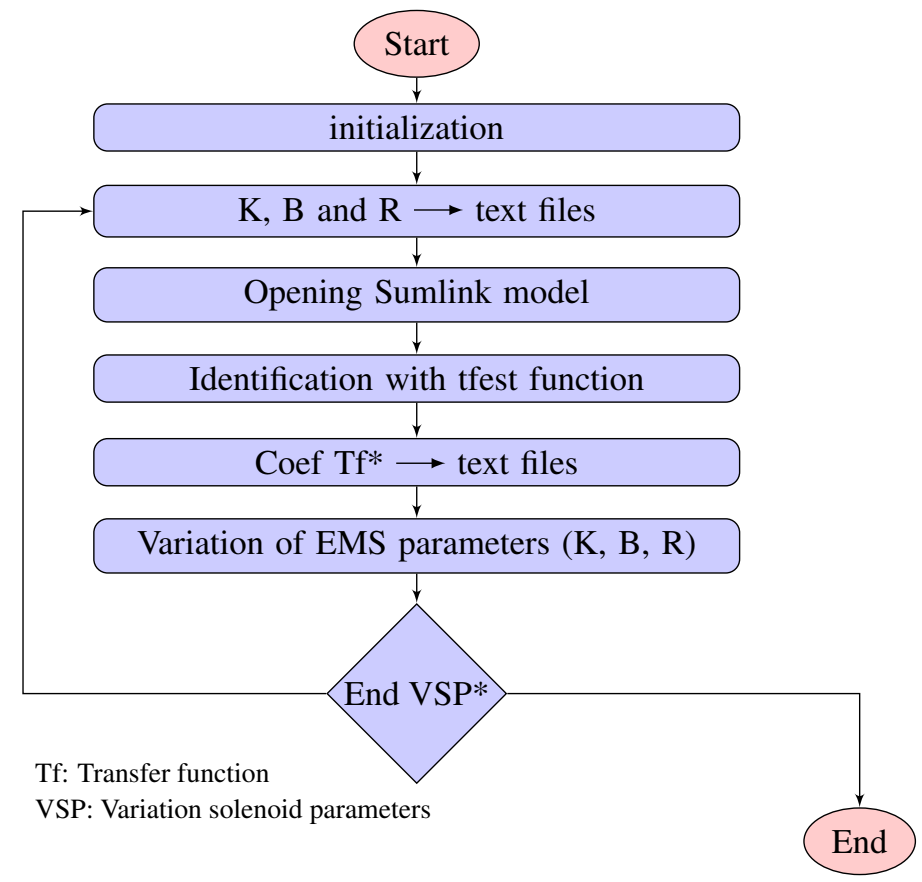

Figure 7. Identification flowchart

\subsubsection{Neural networks training}

The target of neural network is able to identify and predict the solenoid parameters. Data from step response and neural network target are used to search weight (w) and bias (b). Weight and bias are obtained by entering data and target in MATLAB program by using Neural Net fitting toolbox which offers several training functions. The updating of weight and bias values during network training is performed according to the Levenberg-Marquardt optimization which offers faster tracking of system parameter change [41]. The Levenberg-Marquardt algorithm is an efficient and popular damped least squre technique. This algorithm is a combinaison between the steepest gradient descent and the Gauss-Newton algorithms [42]. The activation function at the output of the HLs is the sigmoid function, it delivers a continuously smoother range of values between 0 and 1 and is less expensive in terms of calculation. At the output of the network, the activation function is linear, which creates an output signal proportional to the input. During the searching process of weight and bias, the dataset is subdivided into three percent, $70 \%$ for training, $15 \%$ for the test and $15 \%$ for 
validation. The evaluation of the model is measured using three evaluation performances which are the mean square error (MSE), the coefficient of correlation (R) and error histogram. The optimization technique applied in the ANN model training seeks to optimize the weights and biases of the ANN structure by minimizing rhe mean square error (MSE).

The training results are illustrated in Figure 8(a) which represents the convergent curve of the MSE according to the epochs, error histogram and coefficient of the correlation between the output and the target that respectively illustrated in Figure 8(b) and Figure 8(c).

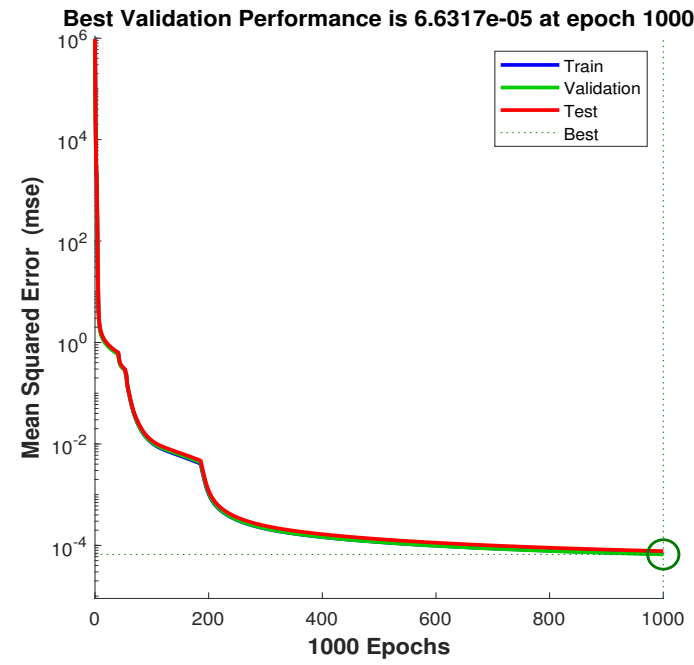

(a)

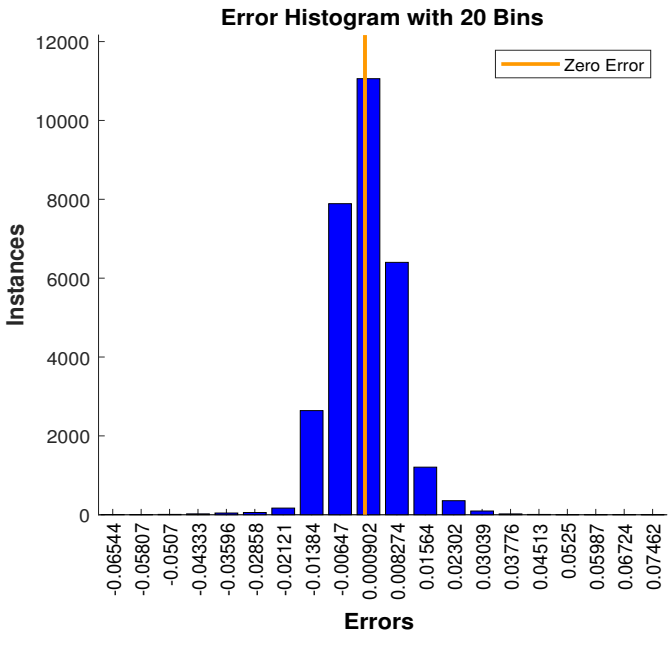

(b)

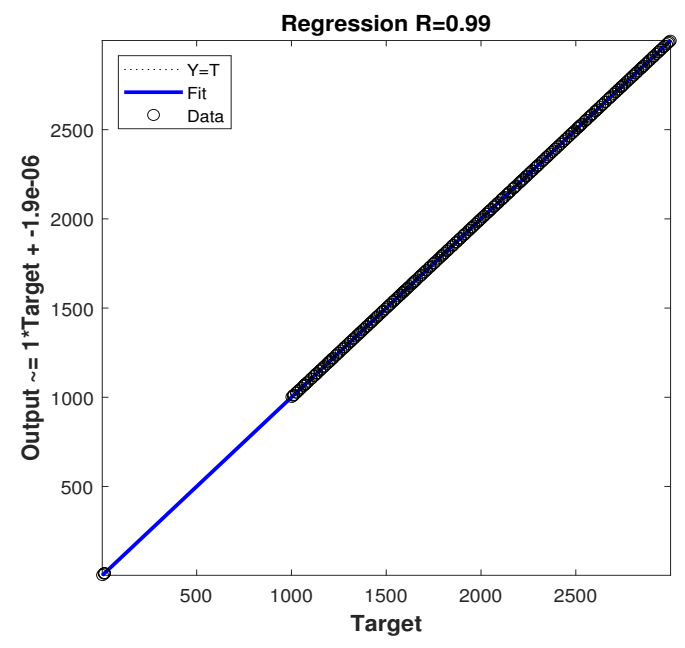

(c)

Figure 8. Training performance, (a) The convergent curve of the MSE, (b) Training error histogram,

(c) Training coefficient of correlation

\subsection{Monitoring result and prediction error}

The prediction and monitoring, of the EMS parameters are achieved through the data acquisition (num, dun2, dun3) from the step response of the optical fiber polarization controller based on EMS. These data are used as inputs of the NN model in order to find the parmeters. The predicted solenoid parameters M, B and K obtained from the transfert function coefficient are illustred in the Table 2. Figure 9 shows the structure of the minotoring process. 
Table 2. Predicted parameters testing result

\begin{tabular}{lcccccr}
\hline & \multicolumn{3}{c}{ NN input } & \multicolumn{3}{c}{ Solenoid parameter } \\
\hline & num & dun2 & dun3 & $\mathrm{K}(\mathrm{N} / \mathrm{m})$ & $\mathrm{B}(\mathrm{Ns} / \mathrm{m})$ & $\mathrm{R}(\Omega)$ \\
Test1 & 74.23 & 18.28 & 5683 & 1101.8 & 3.60 & 8.90 \\
Test2 & 137.86 & 14.03 & 14010 & 2775.5 & 2.76 & 10.40 \\
Test3 & 59.12 & 16.21 & 10871 & 2159.5 & 3.22 & 14.03 \\
\hline
\end{tabular}

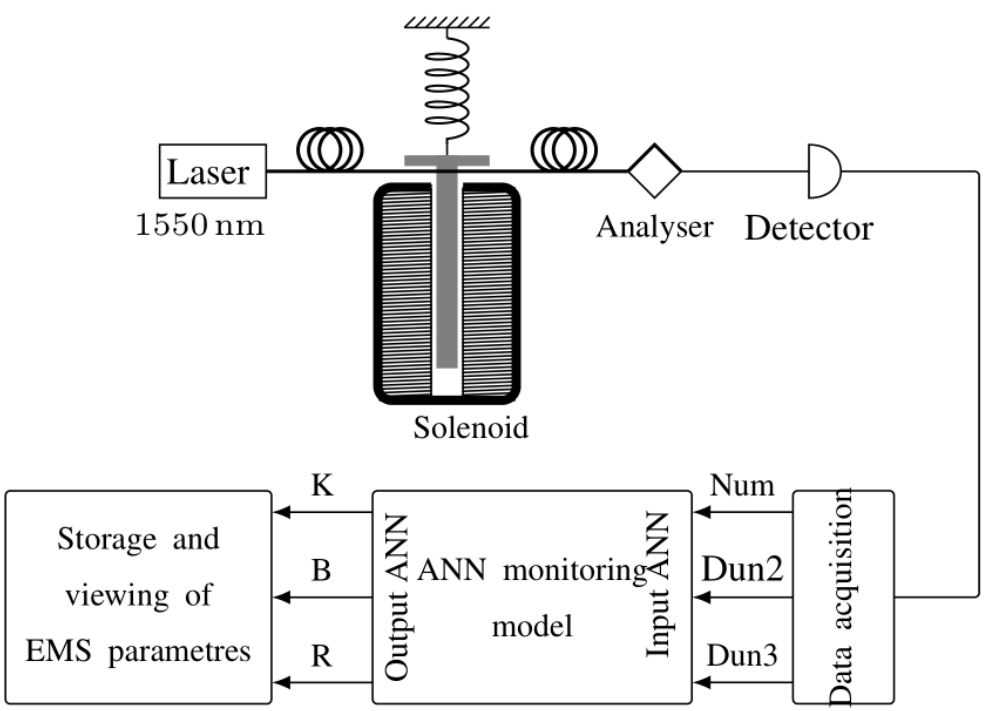

Figure 9. The model architecture for EMS monitoring process

\subsection{Model performance testing}

The evaluation of the model performance is done according to the flowchart of the Figure 10 by using data not utilized for the model training. The parameters found are used as inputs of the Simulink model to find the predicted value of transfer function coefficients. These values are compared to the current values :num, dun 2 and dun 3 to finally calculate the prediction error. The model performance testing structure is illustrated in Figure 11.

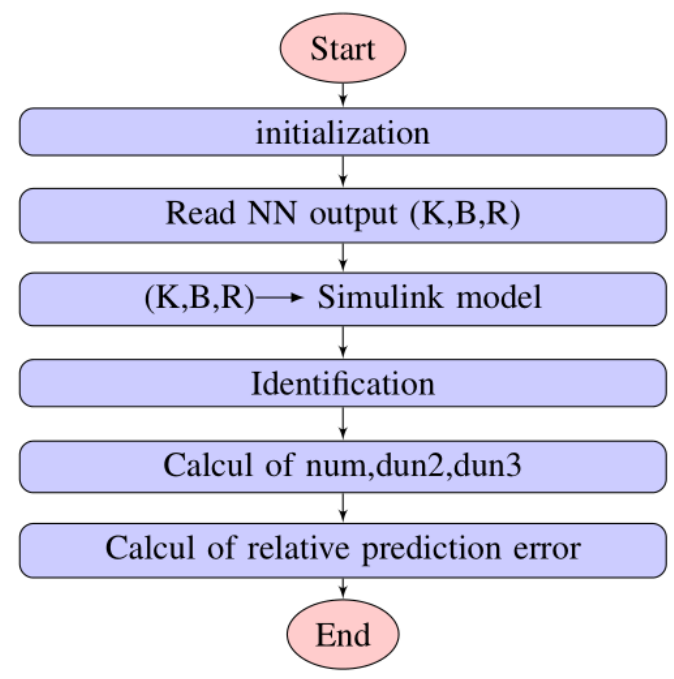

Figure 10. Performance testing flowchart 


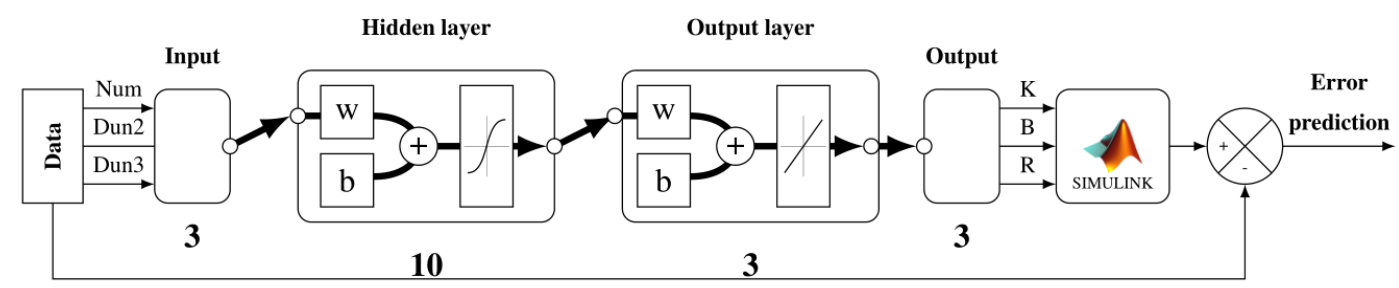

Figure 11. The detail model performance testing

\subsection{Results and discussion}

Table 1 illustrates the result of the mathematical model identification of the system proposed for training using the tfest function for different values of the parameters K, B and R. It is clear from this table that if the stiffness of the spring $\mathrm{K}$, the coefficient of friction $\mathrm{B}$ and the resistance of the solenoid coil $\mathrm{R}$ vary, the coefficients of the transfer function characterizing the model dun 2 and dun 3 also vary, this variation affects the performance of the hydraulic system based on solenoid valve. However, the coefficient dun1 is kept constant it is equal to 1. The Figure 8(a) shows the convergence curve of MSE which illustrates the evolution of the NN training. Moreover, it can be observed from this figure that suitable weights and biases of the NN model are found in the end of the iteration with a better MSE value which is around $6,63 \cdot 10^{-6}$. In addition, the improvoment of the model performance might be realized by increasing the number of iterations (epochs) in order to minimize the MSE. The Gaussian form of the error histogram in Figure 8(b) and the value of coefficient of correlation between outputs and targets during the test illustrated in Figure 8(c) show the high quality of training result.

On the other hand, according to the results obtained from the performance test model of Figure 9 along with those illustrated on the Table 2, it is clear that the predicted values of $\mathrm{K}, \mathrm{B}$ and $\mathrm{R}$ are in evident agreement with the test values obtained by the model of the Figure 11 and which are illustrated in Table 3. What's more, and generally, the training result is less accurate compared to the training, however, from the testing performance given in the Table 3 and the relative prediction error illustrated in Table 4. Thus it is proven that the training performance of Levenberg-Marquardt algorithm is able to control and predict the solenoid parameters, and that the proposed neural network monitoring was successfully implemented.

Table 3. Simulink model testing result

\begin{tabular}{ccccccr}
\hline & \multicolumn{3}{c}{ Solenoid parameters } & \multicolumn{3}{c}{ Simulink model outputs } \\
\hline & $\mathrm{K}(\mathrm{N} / \mathrm{m})$ & $\mathrm{B}(\mathrm{Ns} / \mathrm{m})$ & $\mathrm{R}(\Omega)$ & num & dun2 & dun3 \\
Test1 & 1101.8 & 3.60 & 8.90 & 74.73 & 18.34 & 5684 \\
Test2 & 2775.5 & 2.76 & 10.40 & 137.74 & 14.05 & 14010 \\
Test3 & 2159.5 & 3.22 & 14.03 & 58.97 & 16.21 & 10870 \\
\hline
\end{tabular}

Table 4. The test result prediction error

\begin{tabular}{|c|c|c|c|c|c|c|c|c|c|}
\hline & \multicolumn{3}{|c|}{ Actual values (NN input) } & \multicolumn{3}{|c|}{ Predicted values (simulink model output) } & \multicolumn{3}{|c|}{ Relatif prediction error } \\
\hline & num & dun2 & Dun3 & num & dun2 & dun3 & num & dun2 & Dun3 \\
\hline Test1 & 74.23 & 18.28 & 5883 & 74.73 & 18.34 & 5684 & $0.67 \%$ & $0.30 \%$ & $0.01 \%$ \\
\hline Test2 & 137.86 & 14.03 & 14010 & 137.74 & 14.05 & 14010 & $0.08 \%$ & $0.13 \%$ & $0 \%$ \\
\hline Test3 & 59.12 & 16.21 & 10871 & 58.97 & 16.21 & 10870 & $0 \%$ & $0 \%$ & $0.003 \%$ \\
\hline
\end{tabular}

\section{CONCLUSION}

In this article, a model-based approach for detecting faults in electromagnetic solenoid of valves was proposed. The model is based on ANN coupled with an optical fiber polarization squeezer signal feedback. During a voltage step, the coefficients of the transfer function of the mathematical model are determined from the step response of the actuator model. Then, the parameters of the EMS, selected as the health indices of the solenoid valve, are determined from the ANN model. The results of this model have been verified through simulation on MATLAB/Simulink. The proposed neural networks model has satisfactory performance of prediction and has met the monitoring requirement. Thus, this contribution provides a novel approach on fault diagnostics in hydraulic systems. It consists only of software and optical fiber. Additionally, expensive 
sensors that may reduce the overall reliability, are not required. This method is also applicable to solenoid in other applications, e.g., solenoid actuators in robotics, variable force solenoid in clutch systems, and so on. Future work will not only consist of diagnosing the solenoid valve failure modes by monitoring the EMS parameters, but also through predicting the remaining life.

\section{REFERENCES}

[1] L. Yu and T. N. Chang, "Zero vibration on-off position control of dual solenoid actuator," 33rd Annual Conference of the IEEE Industrial Electronics Society (IECON 2007), Taipei, pp. 775-780, 2007, doi: 10.1109/IECON.2007.44601650.

[2] I. V. Bochkarev, I. V. Bryakin and V. R. Khramshin, "Control of Operational Condition of Electromagnetic Devices of Automation Systems," 2018 International Conference on Industrial Engineering, Applications and Manufacturing (ICIEAM), Moscow, Russia, 2018, pp. 1-6, doi: 10.1109/ICIEAM.2018.8728640.

[3] L. Petit, A. Hassine, J. Terrien, F. Lamarque and C. Prelle, "Development of a Control Module for a Digital Electromagnetic Actuators Array," in IEEE Transactions on Industrial Electronics, vol. 61, no. 9, pp. 4788-4796, Sept. 2014, doi: 10.1109/TIE.2013.2290755.

[4] U. Koch, D. Wiedemann and H. Ulbrich, "Model-Based MIMO State-Space Control of a Car Vibration Test Rig With Four Electromagnetic Actuators for the Tracking of Road Measurements," in IEEE Transactions on Industrial Electronics, vol. 58, no. 12, pp. 5319-5323, Dec. 2011, doi: 10.1109/TIE.2010.2044740.

[5] A. Sosa, D. S. Bollinger, and P. R. Karns, "Performance characterization of a solenoid-type gas valve for the $\mathrm{h}^{-}$ magnetron source at fnal, 2017," AIP Conference Proceedings vol. 1869, 2017, doi: 10.1063/1.4995720.

[6] A. Takai, N. Alanizi, K. Kiguchi and T. Nanayakkara, "Prototyping the flexible solenoid-coil artificial muscle, for exoskeletal robots," 2013 13th International Conference on Control, Automation and Systems (ICCAS 2013), Gwangju, pp. 1046-1051, 2013, doi: 10.1109/ICCAS.2013.6704071.

[7] M. Chen, H. Huang and S. Hung, "A New Design of a Submicropositioner Utilizing Electromagnetic Actuators and Flexure Mechanism,” in IEEE Transactions on Industrial Electronics, vol. 57, no. 1, pp. 96-106, Jan. 2010, doi: 10.1109/TIE.2009.2033091.

[8] L. Chu, Y. Hou, M. Liu, J. Li, Y. Gao and M. Ehsani, "Study on the Dynamic Characteristics of Pneumatic ABS Solenoid Valve for Commercial Vehicle," 2007 IEEE Vehicle Power and Propulsion Conference, Arlington, TX, pp. 641-644, 2007, doi: 10.1109/VPPC.2007.4544201.

[9] S. H. Rumbaugh, M. D. Jones and L. W. Casperson, "Polarization control for coherent fiber-optic systems using nematic liquid crystals," in Journal of Lightwave Technology, vol. 8, no. 3, pp. 459-465, March 1990, doi: $10.1109 / 50.50741$.

[10] Jerry R. Boza, and Kapseong Ro, "Design and Validation of Electro-Hydraulic Pressure-Control Valves for ClosedLoop Controller Implementation," Proceedings of the ASME/BATH 2017 Symposium on Fluid Power and Motion Control. ASME/BATH 2017 Symposium on Fluid Power and Motion Control, Sarasota, Forida, USA. October 16-19, 2017. V001T01A056. ASME. doi: 10.1115/FPMC2017-4311.

[11] X. Tang et al., "A parameter adaptive data-driven approach for remaining useful life prediction of solenoid valves," 2019 IEEE International Conference on Prognostics and Health Management (ICPHM), San Francisco, CA, USA, pp. 1-6, 2019, doi: 10.1109/ICPHM.2019.8819382.

[12] D. Cvetkovic, I. Cosic, and A. Subic, "Improved performance of the electromagnetic fuel injector solenoid actuator using a modelling approach," International Journal of Applied Electromagnetics and Mechanics, vol. 27, no. 4, pp. 251-273, 2008, doi: 10.3233/JAE-2008-939.

[13] M. Kabib, I. Made, L. Batan, B. Pramujati, and A. Sigit, "Modelling and simulation analysis of solenoid valve for spring constant influence to dynamic response," ARPN Journal of Engineering and Applied Sciences, vol. 11, no. 02, pp. 2790-2793, 2016.

[14] X. Tang, M. Xiao, Y. Liang, B. Hu, and L. Zhang, "Application of particle filter technique to online prognostics for solenoid valve," Journal of Intelligent Fuzzy Systems, vol. 35, no. 7 pp. 1-10, 2018, doi: 10.3233/JIFS-169608.

[15] A. di Gaeta, L. Glielmo, V. Giglio, and G. Police, "Modeling of an electromechanical engine valve actuator based on a hybrid analytical-fem approach," in IEEE/ASME Transactions on Mechatronics, vol. 13, no. 6, pp. 625-637, 2008, doi: 10.1109/TMECH.2008.2003469.

[16] E. Ramirez-Laboreo, C. Sagues and S. Llorente, "A New Model of Electromechanical Relays for Predicting the Motion and Electromagnetic Dynamics," in IEEE Transactions on Industry Applications, vol. 52, no. 3, pp. 2545 2553, May-June 2016, doi: 10.1109/TIA.2016.2518120.

[17] E. Ramirez-Laboreo, E. Moya-Lasheras and C. Sagues, "Real-Time Electromagnetic Estimation for Reluctance Actuators," in IEEE Transactions on Industrial Electronics, vol. 66, no. 3, pp. 1952-1961, March 2019, doi: 10.1109/TIE.2018.2838077.

[18] C. Tseng and C.-F. Lin, "Characterisation of solenoid valve failure for electronic diesel fuel injection system of commercial trucks," International Journal of Heavy Vehicle Systems, vol. 13, no. 1, 2006, doi: 
10.1504/IJHVS.2006.010017

[19] Z.-Y. Sun, G.-X. Li, L. Wang, W.-H. Wang, Q.-X. Gao, and J. Wang, "Effects of structure parameters on the static electromagnetic characteristics of solenoid valve for an electronic unit pump," Energy Conversion and Management, vol. 113, pp. 119-130, 2016, doi: 10.1016/j.enconman.2016.01.031.

[20] S. P. Lunge and S. R. Kurode, "Proportional actuator from on off solenoid valve using sliding modes," Proceedings of the 1st International and 16th National Conference on Machines and Mechanisms (iNaCoMM2013), IIT Roorkee, India, Dec 18-20 2013.

[21] A. Zahidi, A. Said, N. Azami, and N. Nasser, "Effect of fiber and solenoid variation parameters on the elements of a corrector pid for electromagnetic fiber squeezer based polarization controller," International Journal of Electrical and Computer Engineering (IJECE), vol. 10, no. 06, pp. 2441-2451, 2020, doi: 10.11591/ijece.v10i3.pp2441-2451.

[22] J. Liniger, S. Stubkier, M. Soltani, and H. C. Pedersen, "Early detection of coil failure in solenoid valves," IEEE/ASME Transactions on Mechatronics, vol. 25, no. 2, pp. 683-693, April 2020, doi: 10.1109/TMECH.2020.2970231.

[23] Mercer JR. "Paper 12: Reliability of Solenoid Valves" Proceedings of the Institution of Mechanical Engineers, Conference Proceedings, vol. 184, no. 2, pp. 89-94, 1969.

[24] S. Angadi, R. Jackson, S.-y. Choe, G. Flowers, J. Suhling, Y.-K. Chang, J.-K. Ham, and J.-i. Bae, "Reliability and life study of hydraulic solenoid valve - part 2 - experimental study," Engineering Failure Analysis, vol. 16. no. 4, pp. 944-963, 2009, doi: 10.1016/j.engfailanal.2008.08.012.

[25] J. Perotti, "Kea-71 smart current signature sensor (scss)," 2010.

[26] H.-H. Tsai and C.-Y. Tseng, "Detecting solenoid valve deterioration in in-use electronic diesel fuel injection control systems," Sensors, vol. 10, no. 8, pp. 7157-69, 2010, doi: 10.3390/s100807157

[27] J. M. Perotti, A. Lucena, C. Ihlefeld, B. Burns, and M. Bassignani, "Washington, dc: U.s. patent and trademark office,” Patent U.S. Patent No. 6,917,203, 2005.

[28] G. Tod, T. Mazaev, K. Eryilmaz, A. P. Ompusunggu, E. Hostens and S. V. Hoecke, "A Convolutional Neural Network Aided Physical Model Improvement for AC Solenoid Valves Diagnosis," 2019 Prognostics and System Health Management Conference (PHM-Paris), Paris, France, pp. 223-227, 2019.

[29] Sokolov, V. Marinochkin, V. Mochalov, and I. Bochkarev, Patent RU patent 1797 099, 1993.

[30] I. Bochkarev, Patent RU patent 2115 151, 1998.

[31] X. Wang, L. Quan, and G. Xiong, "Detecting the position of the moving-iron solenoid by nondisplacement sensor based on parameter identification of flux linkage characteristics," Chinese Journal of Mechanical Engineering, vol. 26, no. 11, pp. 1204-1211, 2013, doi: 10.3901/CJME.2013.06.1204.

[32] Dülk, I., Kovácsházy, T. "Resistance Estimation in Solenoid Actuators by Considering Different Resistances in the PWM Paths", Periodica Polytechnica Electrical Engineering and Computer Science, vol. 58, no. 3, pp. 109-120, 2014, doi: 10.3311/PPee.7353.

[33] T. Trong, "The neural network-based control system of direct current motor driver," International Journal of Electrical and Computer Engineering (IJECE), vol. 9, no. 4, pp. 1445-1452, 2019, doi: 10.11591/ijece.v9i2.pp1445-1452.

[34] H. Widyantara and M. Rivai, "Implementation analog neural network for electronic nose using field programable analog arrays (fpaa)," International Journal of Electrical and Computer Engineering (IJECE), vol. 2, no. 6, pp. 739-747, 2012.

[35] Z. Yusuf, A. P. I. D. N. Wahab, and S. Sahlan, "Neural network model development with soft computing techniques for membrane filtration process," International Journal of Electrical and Computer Engineering, vol. 8, no. 4, pp. 2614-2623, 2018, doi: 10.11591/ijece.v8i4.pp2614-2623.

[36] S. Li, P. Guo, W. Jiang, H. Ding and D. Yu, "Research on response characteristics and parameters optimization of high-speed solenoid valve," 2015 34th Chinese Control Conference (CCC), Hangzhou, pp. 2327-2332, 2015, doi: 10.1109/ChiCC.2015.7259997.

[37] K. Najdek, "Identification of dual-active-bridge converter transfer function," PRZEGLAD ELEKTROTECHNICZNY, 2019, doi: 10.15199/48.2019.03.33.

[38] A. M. Smith, "Single-mode fibre pressure sensitivity," Electronics Letters, vol. 16, no. 20, pp. 773-774, 1980.

[39] C. Li, H. Yang, L. L. Jenkins, R. N. Dean, G. T. Flowers, and J. Y. Hung, "Enhanced-performance control of an electromagnetic solenoid system using a digital controller," IEEE Transactions on Control Systems Technology, vol. 24, no. 5, pp. 1805-1811, Sept. 2016, doi: 10.1109/TCST.2015.2507060.

[40] Dai Dong and Xiaoning Li, "Simulation and experimental research on the response of a novel highpressure pneumatic pilot-operated solenoid valve," in 2012 19th International Conference on Mechatronics and Machine Vision in Practice (M2VIP), Auckland, pp. 480-484, 2012.

[41] W. Zhang, "An extended adaline neural network trained by levenberg-marquardt method for system identification of linear systems," in 2013 25th Chinese Control and Decision Conference (CCDC), pp. 2453-2458, 2013.

[42] Le Duc-Hung, Pham Cong-Kha, Nguyen Thi Thien Trang, and Bui Trong Tu, "Parameter extraction and optimization using levenberg-marquardt algorithm," in 2012 Fourth International Conference on Communications and Electronics (ICCE), Hue, pp. 434-437, 2012, doi: 10.1109/CCE.2012.6315945. 